Article

\title{
Rescuing Suboptimal Patient-Reported Outcome Instrument Data in Clinical Trials: A New Strategy
}

\author{
Chengwu Yang ${ }^{1, *(1)}$ and Kent E. Vrana ${ }^{2}$ \\ 1 Departments of Epidemiology and Health Promotion, College of Dentistry, New York University, \\ New York, NY 10010, USA \\ 2 Department of Pharmacology, College of Medicine, The Pennsylvania State University, \\ Hershey, PA 17033, USA; kev10@psu.edu \\ * Correspondence: chengwu.yang@nyu.edu; Tel.: +1-212-998-9756
}

Received: 12 December 2017; Accepted: 8 March 2018; Published: 20 March 2018

\begin{abstract}
Background: Psychometric instruments such as the Repeated Battery for the Assessment of Neuropsychological Status (RBANS) are commonly used under conditions for which they were not developed or validated. They may then generate troublesome data that could conceal potential findings. Methods: Based on a previously published refinement of the RBANS, we reanalyzed the data on 303 patients from two National Institutes of Health (NIH) trails in Parkinson's disease and contrasted the results using the original versus refined scores. Results: Findings from the original RBANS scores were inconsistent; however, use of the refined scores produced potential findings that were in agreement with independent reports. Conclusion: This study demonstrates that, for negative trials using instrument scores as primary outcomes, it is possible to rescue potential findings. The key to this new strategy is to validate and refine the instrument for the specific disease and conditions under study and then to reanalyze the data. This study offers a demonstration of this new strategy for general approaches.
\end{abstract}

Keywords: patient-reported outcome (PRO); clinical trials; instrument; measurement; scale; psychometrics; clinimetrics; Parkinson's disease; RBANS; factor analysis; placebo effect

\section{Introduction}

Since few health-related psychometric instruments have been "professionally developed" [1], it is common to see suboptimal instrument data in clinical trials. An example was the use of the Repeatable Battery for the Assessment of Neuropsychological Status (RBANS) [2] in two National Institutes of Health (NIH) Exploratory Trials in Parkinson's disease (NET-PD) [3,4]. The RBANS has been popular since its initial publication. According to Web of Science (Thomson Reuters; accessed 12 December 2017), the initial description of RBANS has been cited 472 times. Moreover, it has been translated and used in many other countries such as China [5], Japan [6], and Italy [7]. Its popularity may relate to its brevity. However, the original factor structure of RBANS was theory-driven [2], while multiple subsequent empirical studies have identified optimal factor structures that differ from the original (e.g., [8-14]). This has engendered significant concerns about the validity of the universal use of RBANS to assess cognitive function.

Two NET-PD trials tested four drugs for the treatment of movement impairment in PD: Creatine and Minocycline in FS1, and CoQ10 and GPI1485 in FS-TOO, indicating that Creatine and Minocycline might be promising $[3,4]$. The RBANS was used to assess cognition as a secondary outcome. Because we previously demonstrated that the original RBANS had not been validated for PD, it is not surprising that the RBANS assessments produced equivocal results [13]. Yet, we believe that better use of these problematic, but expensive, data is of critical and practical importance. We therefore set out to 
reanalyze the RBANS data from the two NET-PD trials based on the refined factor structure from our previous study [13], and then to contrast the results with those based on the original factor structure, in hope of rescuing potential findings.

\section{Materials and Methods}

\subsection{Patients}

The two NET-PD trials recruited 858 early untreated PD patients from 42 sites in North America, and randomized 413 participants into six arms. Details can be found in earlier publications $[3,4]$. In total, 339 finished the 12-month follow-up visit. RBANS data were collected at the baseline and 12-month follow-up visit. After the deletion of patients with missing values for the RBANS data and outliers as detected by the Malhanobis distance, 383 and 315 patients remained at baseline and follow-up. Since the change from baseline to follow-up in RBANS scores will be used as the primary outcome, only patients with complete RBANS data at both baseline and follow-up were included in the final analysis of this study. Therefore, 303 patients with complete RBANS data at both baseline and follow-up were analyzed.

\subsection{The RBANS}

The original RBANS has 12 items and offers scores on five cognitive domains [2]. Each of the first four domains is measured by two items, while the last domain is measured by four. In addition, the RBANS offers a total score based on the five domain scores (Figure 1A). However, these six original RBANS scores are neither valid nor reliable in the two NET-PD trials [13].

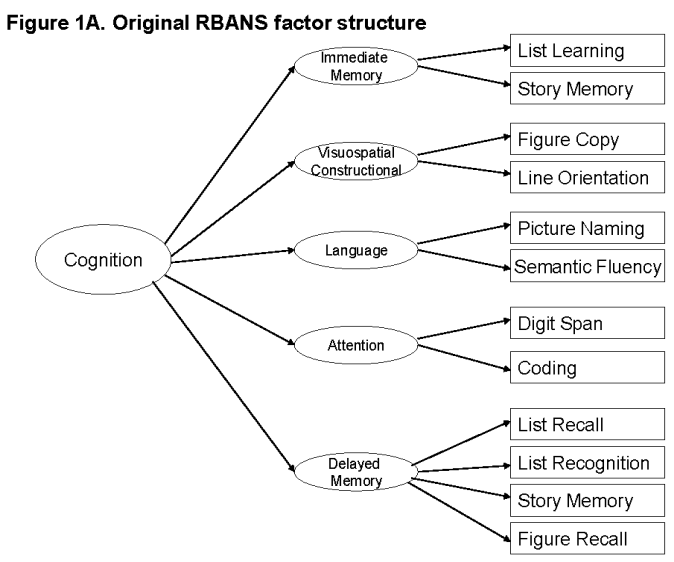

Figure 1B. Refined RBANS factor structure for early PD patients
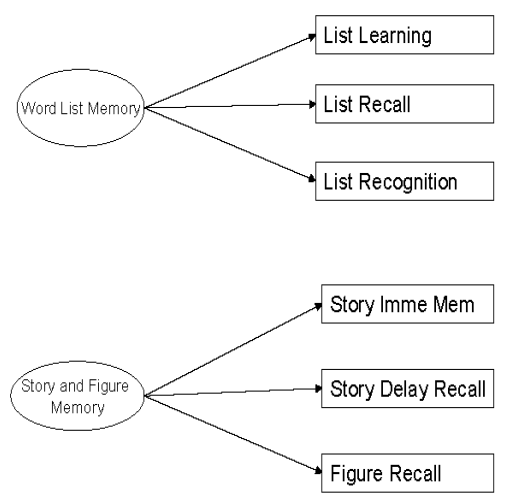

Figure 1. Factor structure of the RBANS: Original vs. refined for early PD patients.

Following psychometric analysis, we created a new instrument that retained half of the assessment items from the original RBANS and reorganized them into two domains [13]. The refined RBANS [13,15] for these trials has six items and offers scores on two new cognitive domains: word list memory (WLM) and story and figure memory (SFM), measured by three items each (Figure 1B). Specifically, six of the original RBANS items were excluded because they had low correlation with each other and the remaining six items [13]. The refined RBANS is valid and reliable for these specific patients, and makes clinical sense [13]. However, a unified total RBANS score based on these two domains was not supported by a two-level factor structure [13].

\subsection{Statistical Analysis}

Data from the two trials were analyzed separately and in parallel. The normality of each variable was checked in order to choose appropriate analysis approaches. Chi-square tests were applied to categorical data, and analysis of variance (ANOVA) or Kruskal-Wallis tests were applied to continuous 
data. Demographic and disease characteristics of the participants at baseline were summarized and compared across the three arms within each of the two trials. Preliminary comparisons of change from baseline on cognitive abilities as measured by the original and refined RBANS, within each of the two trials, were implemented. In comparing the original RBANS with the refined RBANS, we focused on providing consistent changes in cognitive scores. Comparing $p$-values would be flawed given the lack of power in these historical data (inadequate sample size) for assessing these secondary outcome measures [3,4]. Post hoc power analysis and sample size estimation were implemented to address the lack of power and small sample size issues. SPSS (Version 23, IBM Corp., Armonk, NY, USA) and SAS (Version 9.4, SAS Institute Inc., Cary, NC, USA) were used for data preparation and analysis. G*Power (Version 3.1.9.2, University of Düsseldorf, Düsseldorf, Germany) $[16,17]$ ) was used for post hoc power analysis and sample size estimation.

\section{Results}

Demographic and disease characteristics at baseline are summarized in Table 1.

Table 1. Demographic and disease characteristics of participants at baseline.

\begin{tabular}{|c|c|c|c|c|c|}
\hline FS1 $(n=151)$ & & $\begin{array}{l}\text { 1. Creatine } \\
(n=46)\end{array}$ & $\begin{array}{l}\text { 2. Minocycline } \\
(\mathrm{n}=52)\end{array}$ & $\begin{array}{l}\text { 3. Placebo } \\
(n=53)\end{array}$ & $p$-Value \\
\hline Age & Mean \pm SD & $61.0 \pm 10.8$ & $64.1 \pm 10.5$ & $60.7 \pm 9.7$ & 0.11 \\
\hline Education & Mean \pm SD & $15.4 \pm 3.5$ & $15.0 \pm 2.9$ & $15.2 \pm 2.9$ & 0.95 \\
\hline \multirow{2}{*}{ Gender } & Male & $32(69.6 \%)$ & $28(53.9 \%)$ & $34(64.2 \%)$ & \multirow[t]{2}{*}{0.26} \\
\hline & Female & $14(30.4 \%)$ & $24(46.1 \%)$ & $19(35.8 \%)$ & \\
\hline \multirow{2}{*}{ Race } & White & $43(93.5 \%)$ & $51(98.1 \%)$ & $49(92.5 \%)$ & \multirow[t]{2}{*}{0.44} \\
\hline & Others * & $3(6.5 \%)$ & $1(1.9 \%)$ & $4(7.5 \%)$ & \\
\hline \multirow{2}{*}{ Ethnicity } & Not Hispanic or Latino & $45(97.8 \%)$ & $51(98.1 \%)$ & $51(96.2 \%)$ & \multirow[t]{2}{*}{0.99} \\
\hline & Others $t$ & $1(2.2 \%)$ & $1(1.9 \%)$ & $2(3.8 \%)$ & \\
\hline UPDRS Total Score (Parts I-III) & Mean \pm SD & $22.8 \pm 8.9$ & $23.7 \pm 9.2$ & $23.0 \pm 10.3$ & 0.78 \\
\hline Schwab and England Activities of Daily Living scale & Mean \pm SD & $92.9 \pm 5.1$ & $92.5 \pm 6.4$ & $94.5 \pm 4.6$ & 0.17 \\
\hline \multirow{2}{*}{ Tremor Dominant $\ddagger$} & Yes & $21(45.7 \%)$ & $25(48.1 \%)$ & $33(62.3 \%)$ & \multirow[t]{2}{*}{0.19} \\
\hline & No & $25(54.3 \%)$ & $27(51.9 \%)$ & $20(37.7 \%)$ & \\
\hline \multirow{4}{*}{ Hoehn and Yahr Staging } & 0 & 0 & 0 & 0 & \multirow[t]{4}{*}{0.46} \\
\hline & 1 & $27(58.7 \%)$ & $24(46.1 \%)$ & $27(50.9 \%)$ & \\
\hline & 2 & $19(41.3 \%)$ & $28(53.9 \%)$ & $26(49.1 \%)$ & \\
\hline & 3 & 0 & 0 & 0 & \\
\hline FS-TOO $(\mathrm{n}=152)$ & & $\begin{array}{c}\text { 1. CoQ10 } \\
(n=50)\end{array}$ & $\begin{array}{l}\text { 2. Minocycline } \\
(\mathrm{n}=50)\end{array}$ & $\begin{array}{l}\text { 3. Placebo } \\
(\mathrm{n}=52)\end{array}$ & $p$-Value \\
\hline Age & Mean \pm SD & $61.1 \pm 9.2$ & $61.4 \pm 10.9$ & $61.4 \pm 9.2$ & 0.90 \\
\hline Education & Mean \pm SD & $15.6 \pm 3.2$ & $15.4 \pm 3.0$ & $15.4 \pm 2.7$ & 0.97 \\
\hline \multirow{2}{*}{ Gender } & Male & $31(62.0 \%)$ & $32(64.0 \%)$ & $38(73.1 \%)$ & \multirow[t]{2}{*}{0.45} \\
\hline & Female & $19(38.0 \%)$ & $18(36.0 \%)$ & $14(26.9 \%)$ & \\
\hline \multirow{2}{*}{ Race } & White & $47(94.0 \%)$ & $49(98.0 \%)$ & 47 (90.4\%) & \multirow[t]{2}{*}{0.30} \\
\hline & Others * & $3(6.0 \%)$ & $1(2.0 \%)$ & $5(9.6 \%)$ & \\
\hline \multirow{2}{*}{ Ethnicity } & Not Hispanic or Latino & $50(100.0 \%)$ & $48(96.0 \%)$ & $52(100.0 \%)$ & \multirow[t]{2}{*}{0.21} \\
\hline & Others $t$ & 0 & $2(4.0 \%)$ & 0 & \\
\hline UPDRS Total Score (Parts I-III) & Mean \pm SD & $22.0 \pm 9.6$ & $20.8 \pm 8.8$ & $22.2 \pm 9.1$ & 0.67 \\
\hline Schwab and England Activities of Daily Living scale & Mean \pm SD & $92.9 \pm 5.7$ & $93.8 \pm 4.4$ & $93.1 \pm 4.9$ & 0.78 \\
\hline \multirow[t]{2}{*}{ Tremor Dominant $\ddagger$} & Yes & $26(52.0 \%)$ & $31(62.0 \%)$ & $27(51.9 \%)$ & \multirow[t]{2}{*}{0.50} \\
\hline & No & $24(48.0 \%)$ & $19(38.0 \%)$ & $25(48.1 \%)$ & \\
\hline \multirow{4}{*}{ Hoehn and Yahr Staging } & 0 & $1(2.0 \%)$ & 0 & 0 & \multirow[t]{4}{*}{0.96} \\
\hline & 1 & $27(54.0 \%)$ & $28(56.0 \%)$ & $30(57.7 \%)$ & \\
\hline & 2 & $21(42.0 \%)$ & $22(44.0 \%)$ & $22(42.3 \%)$ & \\
\hline & 3 & $1(2.0 \%)$ & 0 & 0 & \\
\hline
\end{tabular}

* Other races include Indian, Alaska Native, Asian, Black/African American, more than one race, and unknown or not reported. + Other ethnicities include Hispanic or Latino, and unknown or not reported. ‡ A PD patient was determined to be tremor dominant or not by using the method described by Jankovic et al. (1990) [18]. UPDRS: unified Parkinson's disease rating scale. 
No statistically significant difference was detected at any of these characteristics among the two treatment groups and one placebo group within each of the trials, and characteristics are very similar within each of the two trials.

Preliminary comparisons of change from baseline on cognitive abilities are summarized in Table 2.

Table 2. Preliminary comparisons of change from baseline on cognitive abilities among treatment groups using the Repeated Battery for the Assessment of Neuropsychological Status (RBANS) scores: original vs. refined.

\begin{tabular}{|c|c|c|c|c|c|c|c|c|c|}
\hline \multirow{10}{*}{$\begin{array}{c}\text { FS1 } \\
(n=151)\end{array}$} & \multirow{2}{*}{\multicolumn{2}{|c|}{ RBANS Scores }} & \multicolumn{2}{|c|}{ Creatine $(n=46)$} & \multicolumn{2}{|c|}{ Minocycline $(n=52)$} & \multicolumn{2}{|c|}{ Placebo $(n=53)$} & \multirow{2}{*}{$p$-Value } \\
\hline & & & Mean & SD & Mean & SD & Mean & SD & \\
\hline & \multirow[t]{6}{*}{ Original } & IM & 5.93 & 12.79 & 4.63 & 12.18 & 3.77 & 11.95 & 0.86 \\
\hline & & VC & -2.91 & 14.43 & -7.29 & 18.37 & -2.28 & 17.39 & 0.34 \\
\hline & & $\mathrm{La}$ & -1.22 & 10.52 & -2.94 & 9.33 & 0.53 & 9.34 & 0.09 \\
\hline & & Att & 0.63 & 13.17 & -1.38 & 14.43 & -0.94 & 12.48 & 0.87 \\
\hline & & $\mathrm{DM}$ & 2.09 & 13.49 & -0.52 & 11.45 & -2.28 & 14.28 & 0.26 \\
\hline & & Total & 1.39 & 9.77 & -2.81 & 10.04 & -0.94 & 9.81 & 0.14 \\
\hline & \multirow[t]{2}{*}{ Refined } & WLM & 3.00 & 5.52 & 0.75 & 5.55 & 1.25 & 5.95 & 0.15 \\
\hline & & SFM & 1.11 & 7.91 & 0.87 & 7.36 & 0.11 & 6.25 & 0.95 \\
\hline \multirow{10}{*}{$\begin{array}{l}\text { FS-TOO } \\
(n=152)\end{array}$} & \multirow{2}{*}{\multicolumn{2}{|c|}{ RBANS Score }} & \multicolumn{2}{|c|}{ CoQ10 (n = 50) } & \multicolumn{2}{|c|}{ GPI1485 $(n=50)$} & \multicolumn{2}{|c|}{ Placebo $(n=52)$} & $p$-Value \\
\hline & & & Mean & SD & Mean & SD & Mean & SD & \\
\hline & \multirow[t]{6}{*}{ Original } & IM & 0.60 & 10.24 & 0.18 & 10.41 & 0.19 & 14.98 & 0.82 \\
\hline & & VC & -0.96 & 14.45 & -5.72 & 16.90 & -1.94 & 14.76 & 0.50 \\
\hline & & $\mathrm{La}$ & 1.44 & 9.55 & -0.16 & 8.11 & -1.60 & 8.38 & 0.13 \\
\hline & & Att & -0.02 & 13.09 & 0.02 & 12.90 & 1.65 & 13.69 & 0.71 \\
\hline & & $\mathrm{DM}$ & 4.78 & 14.23 & 1.18 & 20.24 & 0.37 & 14.63 & 0.51 \\
\hline & & Total & 2.14 & 9.82 & -1.16 & 10.43 & -0.52 & 10.87 & 0.24 \\
\hline & \multirow[t]{2}{*}{ Refined } & WLM & 0.10 & 4.71 & 0.60 & 4.92 & 0.52 & 6.12 & 0.80 \\
\hline & & SFM & 1.52 & 5.66 & -0.12 & 6.86 & -0.83 & 5.36 & 0.13 \\
\hline
\end{tabular}

None of the pairwise comparisons of the refined RBANS scores was statistically significant ( $p$-value not shown), mainly due to the small sample size; IM: Immediate Memory; VC: Visuospatial/Constructional; La: Language; Att: Attention; DM: Delayed Memory; WLM: Word List Memory; SFM: Story and Figure Memory [13].

When cognition is measured by the original six RBANS scores, there is no consistent trend over time. Out of the 36 changes, 17 (47.2\%) are increased, 19 (52.8\%) are decreased. Moreover, none of the six groups show consistency in the direction of change among the original six RBANS scores. For example, in the Creatine group, while four original RBANS scores show increases, the other two show decreases. In contrast, when cognition is measured by the two refined RBANS scores, the trend over time is much more consistent. Out of the 12 changes on refined RBANS scores, 10 (83.3\%) show increases, and only two show a decrease, but both within one unit $(-0.12,-0.83)$. None of the differences among groups, in change from baseline on either of the RBANS scores, is statistically significant $(p>0.05)$, because the study was underpowered. Clearly, however, the refined RBANS provides much more consistent data trends, and will therefore give greater insight into treatment outcomes.

Post hoc power analysis indicated that, in order to have $80 \%$ power to detect the difference shown on WLM between Creatine and Placebo (3.00 vs. 1.25, Cohen's $\mathrm{d}=0.30$ [19]), 178 patients per group would be needed. Given the current sample size of 50 per group, the statistical power to detect this difference was $31 \%$. Since the effect size of Creatine on WLM was the largest, post hoc analysis on others would result in a larger required sample size or would show lower power at the current sample size.

\section{Discussion}

Given that suboptimal instrument data are commonly utilized in clinical trials, it is of great practical importance to better utilize these troublesome and expensive data in hope of rescuing important potential findings. This study offers a template for rescuing efforts through reanalyzing 
existing suboptimal instrument data. Findings from the two NET-PD trials that employed the unified Parkinson's disease rating scale (UPDRS) scores as the primary outcome indicated that two (Creatine, Minocycline) of the four tested drugs may be beneficial for PD patients [3], while the other two (CoQ10, GPI1485) may not [4]. Results from the present study indicate that, while using the original six RBANS scores showed no benefit of the treatments on cognitive ability as a secondary outcome, the use of the two refined RBANS scores may have produced a positive outcome had the sample sizes been larger (Table 2).

The inconsistencies in the trend among the original six RBANS scores are very troublesome. It is not easy to explain why a drug can help improve some cognitive abilities while impairing others (Table 2). This observation strengthens the conclusion that the RBANS was neither valid nor reliable in the two NET-PD trials studied here [13]. In contrast, the refined RBANS scores offer much more consistency in the trend, albeit in the absence of statistical significance. Most of the changes from baseline are increasing, indicating that the treatment is increasing each of the cognitive abilities. The increased outcomes in cognitive assessments offer more practical support for the validity and reliability of the refined RBANS in both trials [13].

Another advantage of the refined versus the original RBANS is indicated by the big differences in the standard deviations (SD) of the changes from baseline (Table 2). When using the original RBANS, the SDs are huge (e.g., for Creatine, Att has a mean of 0.65 and an SD of 13.17). However, after refinement, the SDs dropped substantially (e.g., for Creatine, WLM has a mean of 3.00 and an SD of 5.52 .

Placebo effect [20] on the two refined RBANS scores is evidenced by the three increases in the two placebo groups. Participating in a clinical trial and receiving some kind of treatment may help patients feel better and can improve their cognition. However, these placebo effects are smaller than potential true treatment effects.

Lack of power due to the small sample size is the primary reason for potentially physiologically important, but statistically negative results. Had the sample size been large enough, these results should also be statistically significant. Future studies with appropriate sample size are therefore warranted.

Clearly, there are important limitations in this analysis related to statistical power. Take, for example, the observed differences on WLM between Creatine and Placebo in FS1 (details in Table 2). The observed difference was 3.00 for Creatine and 1.25 for Placebo, with Cohen's d as 0.30, which was between "small" and "medium" [19]. That is to say, the difference was clearly "clinically significant". However, due to the small sample size (46 in Creatine, 53 in Placebo, 99 total), the $p$-value was 0.15, and the difference was "statistically insignificant". This is a typical scenario when a study finding is "clinically significant", but "statistically insignificant", since the sample size is not big enough. However, clinical significance should be an important deciding factor for medical studies, not simply statistical significance, because " $p$-values do not measure evidence" [20] (p. 619). In addition, recent reports have re-emphasized the severe issue of $p$-driven research (e.g., [21]), including the American Statistical Association (ASA) statement on $p$-values [22]. What the present studies emphasize, however, is that a properly designed and validated instrument, combined with an appropriate sample size, can provide both clinical and statistical significance.

In clinical trials that use psychometric instruments, it is critical to validate or even refine the instruments for data collected before any formal statistical analysis. This is because most instruments are "not professionally developed" [1], and instrument validation is an "ongoing process" [23]. No instrument should be claimed to be "already validated"; rather, assessment instruments should be validated for the disease and population under study. Another good example appears a recent review on oral health-related quality of life (OHRQoL) instruments [24]. Other recent literature re-emphasizes the importance of sound psychometric properties of an instrument [25-27].

For negative trials that used instrument scores as primary outcomes, the present findings offer a path to rescuing potential findings: validating and refining the instruments and then reanalyzing the 
data based on the refined instrument scores. Our study offers a demonstration of the new strategy for this type of promising effort.

\section{Conclusions}

This study demonstrates that, for negative trials using instrument scores as primary outcomes, it is possible to rescue potential findings. The key to this new strategy is to validate and refine the instrument for the specific disease and conditions under study and then to reanalyze the data. This study offers a demonstration of this new strategy for general approaches.

Acknowledgments: This study was sponsored, in part, by the NIH (National Institute of Neurological Disorders and Stroke), U01NS043127, U01NS043128, and U10NS44415 through 44555, and by the National Institute on Aging (NIA) Resource Centers for Minority Aging Research (RCMAR) Grant 3P30 AG021677-02S1. We thank the NINDS NET-PD Investigators for the high-quality data collected in NET-PD FS1 and FS-TOO. The authors wish to acknowledge Barbara C. Tilley and Jay S. Schneider for their contributions to a poster presentation made at the 35th Annual Meeting of the Society for Clinical Trials (SCT), Philadelphia, Pennsylvania, 18-21 May 2014. Part of the materials in this study are contained within that poster.

Author Contributions: C.Y. conceived and designed the study, analyzed the data, and wrote the paper; K.E.V. contributed to conceiving the study and interpreting the results, and performed critical revision of the drafts.

Conflicts of Interest: The authors declare no conflicts of interest.

\section{References}

1. Teresi, J.A.; Fleishman, J.A. Differential item functioning and health assessment. Qual. Life Res. 2007, 16 (Suppl. 1), 33-42. [CrossRef] [PubMed]

2. Randolph, C.; Tierney, M.C.; Mohr, E.; Chase, T.N. The Repeatable Battery for the Assessment of Neuropsychological Status (RBANS): Preliminary Clinical Validity. J. Clin. Exp. Neuropsychol. 1998, 20, 310-319. [CrossRef] [PubMed]

3. NINDS NET-PD Investigators. A randomized, double-blind, futility clinical trial of creatine and minocycline in early Parkinson disease. Neurology 2006, 66, 664-671.

4. NINDS NET-PD Investigators. A randomized clinical trial of coenzyme Q10 and GPI-1485 in early Parkinson disease. Neurology 2007, 68, 20-28.

5. Xu, Y.; Lu, Z. Assessment and treatment of cognitive dysfunction in schizophrenia patient. World Clin. Drugs 2016, 37, 8-12.

6. Nakatsu, D.; Fukuhara, T.; Chaytor, N.S.; Phatak, V.S.; Avellino, A.M. Repeatable Battery for the Assessment of Neuropsychological Status (RBANS) as a Cognitive Evaluation Tool for Patients with Normal Pressure Hydrocephalus. Neurol. Med. Chir. 2016, 56, 51-61. [CrossRef] [PubMed]

7. Costaggiu, D.; Ortu, F.; Pinna, E.; Serchisu, L.; di Martino, M.L.; Manconi, P.E.; Mandas, A. RBANS: A valid tool for cognitive assessment of HIV-infected people on cART. G. Gerontol. 2015, 63, 268-273.

8. Carlozzi, N.E.; Horner, M.D.; Yang, C.; Tilley, B.C. Factor Analysis of the Repeatable Battery for the Assessment of Neuropsychological Status. Appl. Neuropsychol. 2008, 15, 274-279. [CrossRef] [PubMed]

9. Duff, K.; Langbehn, D.R.; Schoenberg, M.R.; Moser, D.J.; Baade, L.E.; Mold, J.; Scott, J.G.; Adams, R.L. Examining the Repeatable Battery for the Assessment of Neuropsychological Status: Factor Analytic Studies in an Elderly Sample. Am. J. Geriatr. Psychiatry 2006, 14, 976-979. [CrossRef] [PubMed]

10. Garcia, C.; Leahy, B.; Corradi, K.; Forchetti, C. Component Structure of the Repeatable Battery for the Assessment of Neuropsychological Status in Dementia. Arch. Clin. Neuropsychol. 2008, 23, 63-72. [CrossRef] [PubMed]

11. Torrence, N.D.; John, S.E.; Gavett, B.E.; O’Bryant, S.E. An Empirical Comparison of Competing Factor Structures for the Repeatable Battery for the Assessment of Neuropsychological Status: A Project Frontier Study. Arch. Clin. Neuropsychol. 2016, 31, 88-96. [CrossRef] [PubMed]

12. Vogt, E.M.; Prichett, G.D.; Hoelzle, J.B. Invariant Two-Component Structure of the Repeatable Battery for the Assessment of Neuropsychological Status (RBANS). Appl. Neuropsychol. Adult 2017, 24, 50-64. [CrossRef] [PubMed] 
13. Yang, C.; Garrett-Mayer, E.; Schneider, J.S.; Gollomp, S.M.; Tilley, B.C. Repeatable battery for assessment of neuropsychological status in early Parkinson's disease. Mov. Disord. 2009, 24, 1453-1460. [CrossRef] [PubMed]

14. Wilde, M. The validity of the repeatable battery of neuropsychological status in acute stroke. Clin. Neuropsychol. 2006, 20, 702-715. [CrossRef] [PubMed]

15. Yang, C.; Schneider, J.; Tilley, B. Fix the left side of an equation: How to rescue positive findings from a clinical trial that used scales if they were wrong?-Illustration with an example. In Proceedings of the 35th Annual Meeting of the Society for Clinical Trials (SCT), Philadelphia, PA, USA, 18-21 May 2014.

16. Faul, F.; Erdfelder, E.; Lang, A.-G.; Buchner, A. G*Power 3: A flexible statistical power analysis program for the social, behavioral, and biomedical sciences. Behav. Res. Methods 2007, 39, 175-191. [CrossRef] [PubMed]

17. Faul, F.; Erdfelder, E.; Buchner, A.; Lang, A.G. Statistical power analyses using G*Power 3.1: Tests for correlation and regression analyses. Behav. Res. Methods 2009, 41, 1149-1160. [CrossRef] [PubMed]

18. Jankovic, J.; McDermott, M.; Carter, J.; Gauthier, S.; Goetz, C.; Golbe, L.; Huber, S.; Koller, W.; Olanow, C.; Shoulson, I.; Stern, M. Variable expression of Parkinson's disease: A base-line analysis of the DAT ATOP cohort. Neurology 1990, 40, 1529-1534. [CrossRef] [PubMed]

19. Cohen, J. Statistical power analysis. Curr. Dir. Psychol. Sci. 1992, 1, 98-101. [CrossRef]

20. Piantadosi, S. Clinical Trials: A Methodologic Perspective, 3th ed.; John Wiley \& Sons: Hoboken, NJ, USA, 2017.

21. Nuzzo, R. Scientific method: Statistical errors. Nature 2014, 506, 150-152. [CrossRef] [PubMed]

22. Ronald, L.W.; Lazar, N.A. The ASA's Statement on $p$-Values: Context, Process, and Purpose. Am. Stat. 2016, 70, 129-133. [CrossRef]

23. Zumbo, B.D. Validity: Foundational Issues and Statistical Methodology. Handb. Stat. 2006, 26, 45-79.

24. Haag, D.G.; Peres, K.G.; Balasubramanian, M.; Brennan, D.S. Oral Conditions and Health-Related Quality of Life: A Systematic Review. J. Dent. Res. 2017, 96, 864-874. [CrossRef] [PubMed]

25. Zumbo, B.D.; Chan, E.K.H. (Eds.) Validity and Validation in Social, Behavioral, and Health Sciences; Social Indicators Research Series; Springer: New York, NY, USA, 2014.

26. American Academy of Clinical Neuropsychology (AACN). AACN practice guidelines for neuropsychological assessment and consultation. Clin. Neuropsychol. 2007, 21, 209-231.

27. American Educational Research Association (AERA); American Psychological Association (APA); National Council on Measurement in Education (NCME). Standards for Educational and Psychological Testing; AERA: Washington, DC, USA, 2014. 